\title{
Opioid Abuse: A Detailed Examination of Cost Drivers over a 24-Month Follow-up Period
}

\author{
Lauren M. Scarpati, PhD; Noam Y. Kirson, PhD; Zitong B. Jia, BS;
} Jody Wen, BS; and Jaren Howard, PharmD

\begin{abstract}
BACKGROUND: Previous work has documented the considerable economic burden associated with opioid abuse, dependence, and overdose/poisoning (hereafter, "abuse"). Recent analyses have provided insights into the trajectory and drivers of the excess costs of abuse both before and after diagnosis, showing the important role of other substance abuse, mental health issues, and painful conditions.
\end{abstract}

OBJECTIVE: To build on the recently published study by Kirson et al. (2017) and extend its findings by (a) evaluating the trajectory of excess costs of abuse for an additional year after an incident abuse diagnosis and (b) exploring the diagnosis-level drivers of excess costs over time in greater detail.

METHODS: Using administrative medical and pharmacy claims, which included payment amounts, for beneficiaries covered by large self-insured companies throughout the United States, abusers were matched to controls using the same methods as in Kirson et al. Excess health care costs were assessed over a 24-month follow-up period, which comprised the 6 months before the initial abuse diagnosis and the 18 months after. Drivers of excess costs were then evaluated by diagnosis (grouped at the 3-digit ICD-9-CM level).

RESULTS: This study analyzed 9,345 matched pairs of abusers and nonabusers. Similar to the previous study, mean per-patient excess health care costs were found to rise considerably leading up to and shortly after the incident diagnosis of abuse, reaching $\$ 15,764$ over the first half of the follow-up period. Over the newly extended follow-up period (months 6 to 18 after diagnosis), excess costs remained elevated $(\$ 7,346)$ and did not return to baseline levels. Over time, an increasing share of excess costs was observed for outpatient services and prescription drug use, relative to acute care settings. A detailed examination of cost drivers suggested elevated costs in several clinical categories (e.g., gastrointestinal, respiratory conditions) beyond those previously identified.

CONCLUSIONS: This research finds that the excess medical costs of abuse extend for at least 1 more year than previously documented, reflecting the need for considerable follow-up care over time. The identification of several other clinical categories with elevated excess costs suggests important areas for future research into the interaction of opioid abuse with the management of other conditions.

J Manag Care Spec Pharm. 2017;23(11):1110-15

Copyright @ 2017, Academy of Managed Care Pharmacy. All rights reserved.

\section{What is already known about this subject}

Opioid abuse imposes a substantial burden on payers.

Excess costs begin rising before the formal, incident diagnosis of abuse; peak during the month of the diagnosis; then begin to decline shortly after.

Analysis of primary cost drivers suggests that abuse patients incur substantial excess costs due to opioid abuse, nonopioid drug/alcohol abuse, mental health issues, and painful conditions before their first diagnosis of opioid abuse.

\section{What this study adds}

Excess medical costs associated with opioid abuse remain elevated for at least 1 more year after diagnosis than previously documented for members of self-funded companies.

Patients receive follow-up care for an abuse issue well after the initial diagnosis, with a greater share of care being provided in an outpatient setting over time.

A more detailed examination of diagnosis-level drivers of excess costs suggests that other disease areas beyond those previously identified also contribute to the excess costs of opioid abuse.

$\top$ n a recent study by Kirson et al. (2017), ${ }^{1}$ we provided updated estimates of the excess costs associated with opioid abuse, dependence, overdose, and poisoning (hereafter, "abuse") over 1 year centered on an incident abuse diagnosis. That study documented that excess costs began increasing 5 months before the initial diagnosis, driven to a large degree by medical resource use associated with nonopioid drug and alcohol abuse. Diagnosis of opioid abuse was associated with a substantial spike in excess costs, and costs remained elevated relative to baseline for 6 months following the diagnosis. Excess costs were driven by medical resource use associated with treating opioid abuse, as well as nonopioid drug and alcohol abuse, mental health issues, and painful conditions. The results pointed to the complex nature of opioid abuse, which often occurs in the context of these other health issues.

The previous study, however, considered a fairly short follow-up period, and it is unclear how the trajectory of medical costs of opioid abuse evolves over a longer period. In addition, while the previous study demonstrated the benefits of describing the primary cost drivers among abusers as a way to better 
understand the complex nature of care for these patients, it considered a relatively short list of such drivers.

The objective of the current work was 2-fold: to (1) evaluate the average monthly incremental costs of opioid abuse for an additional year after diagnosis to more fully understand the trajectory of excess costs and (2) more deeply explore the diagnosis-level drivers of these excess costs.

\section{Methods}

A full description of the data and methods used can be found in the original analysis. ${ }^{1}$ We used de-identified administrative medical and pharmacy claims data from OptumHealth Care Solutions. The data include medical claims (date of service, diagnoses received, procedures performed, place of service, and payment amounts); pharmacy claims (fill dates, national drug codes, and payment amounts); and eligibility information (patient demographics and enrollment history). Two mutually exclusive cohorts, incident opioid "abusers" and "nonabusers," were defined. All patients, both abusers and nonabusers, were required to be aged 18-64 years and continuously eligible in non-health maintenance organization plans throughout the study period. Specifically, patients were required to be continuously covered during the 12 months before the abuse diagnosis; we did not impose after diagnosis eligibility requirements.

For the abuser cohort, we excluded patients who had only a single outpatient diagnosis claim of opioid dependence and who had received a prescription opioid from a health care provider in the previous 6 months. To further ensure that the diagnosis of opioid use disorder was an incident diagnosis, we excluded patients prescribed medications whose primary purpose is the treatment of opioid dependence: methadone or buprenorphine (excluding transdermal patches) during the baseline period.

Opioid abuse, dependence, and overdose/poisoning were identified in the medical claims using International Classification of Diseases, Ninth Revision, Clinical Modification (ICD-9-CM) codes (a list of codes can be found in Kirson et al.), with abusers' index dates defined using the first code for opioid abuse during January 2012-March 2015. The incident date for nonabuser controls was a randomly selected medical claim occurring during the same period.

Each individual was observed over a 12- to 30-month period (the "study period"). As in the previous study, the baseline period was defined as the 6-month period starting 12 months before the index date. The follow-up period, over which costs were measured, comprised the 24-month period beginning 6 months before the abuse diagnosis and extending up to 18 months after diagnosis. The 6-month period directly preceding the index date was included in the follow-up period, as excess costs among opioid abusers are observed even before diagnosis. ${ }^{2}$ The follow-up period was expanded beyond that used in our previous study to include an additional year after diagnosis: Each individual was observed for up to 18 months after diagnosis, compared with up to 6 months after diagnosis in Kirson et al. A diagram of the abbreviated, 18-month study period can be found in Figure 1 of Kirson et al. ${ }^{1}$

Propensity score matching was used to match the abusers and nonabusers to account for confounding factors associated with health care costs. We used an extensive list of demographic, clinical, and health care resource utilization-related variables observed over the baseline period in the propensity score model. ${ }^{1}$ Length of available follow-up, as determined by patients' continuous eligibility, was included in the model as well to ensure that the 2 cohorts had similar availability of followup data. Specifically, given the extended study period duration compared with that of Kirson et al., abusers and controls were re-matched using an expanded set of covariates that included additional indicator variables for the additional possible months of follow-up. Baseline characteristics for the postmatched samples were computed to verify the quality of the match.

We compared abusers and nonabusers in terms of health care costs within 1- and 6-month increments of the study period to compute excess costs (2015 U.S. dollars). Here, health care costs refer to the insurer-paid amounts reflected on the medical and prescription drug claims. Medical costs were categorized by place of service: inpatient; emergency department (ED); outpatient/other (e.g., skilled nursing facilities); and rehabilitation facilities.

The costs estimated at the monthly level serve to describe the excess cost trajectory over time, rather than as a measure of the overall burden to payers. Note that, as patients were not required to have continuous eligibility post-index, the trend in the monthly time-series would be strongly affected by inclusion of zeroes for missing patients. As such, mean monthly costs after diagnosis excluded patients in months for which they no longer had continuous eligibility, as treating their missing cost values as zeroes would conflate changes in costs with loss to follow-up.

On the other hand, mean costs defined within the three 6-month periods after diagnosis are intended to estimate the overall burden from the payer perspective. There, we included patients in months for which they no longer had continuous eligibility, as treating costs for patients who dropped out of the sample as zeroes, rather than missing values, for some portion of the follow-up period yields a more accurate picture of average per-patient excess costs faced by payers. Specifically, these patients may be costlier, as losing coverage may be due to a more severe abuse problem. Including these patients allows us to better capture these patients' costs.

In Kirson et al., the top 15 diagnoses contributing to the excess medical costs were computed by 3-digit ICD-9-CM groupings. We build on that work, computing the top 50 diagnoses in terms of contribution to excess medical costs (also grouped by 3-digit ICD-9-CM diagnosis codes). This detailed list was then binned into larger categories for ease of exposition (the full table of diagnosis-level cost drivers is available upon request). 


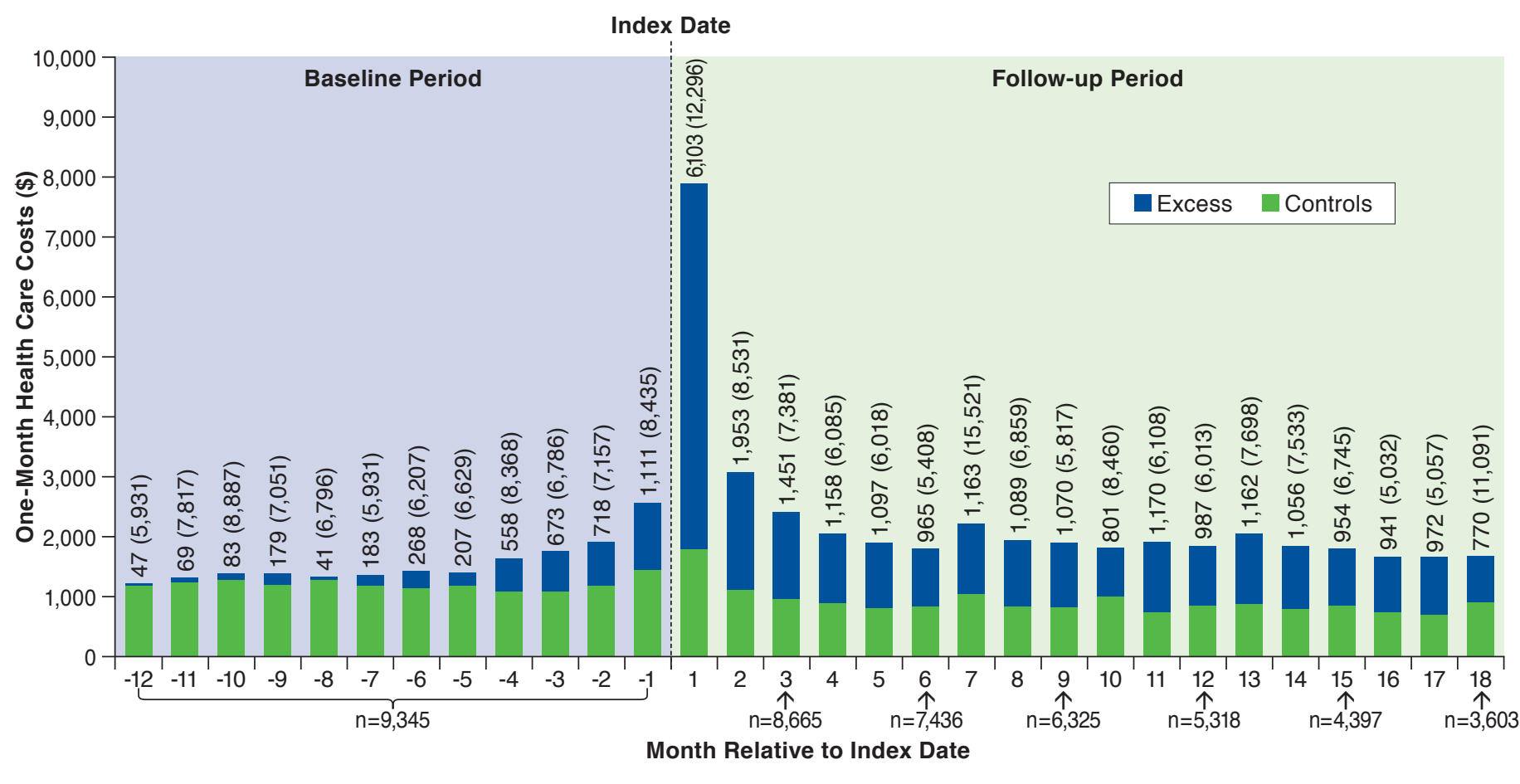

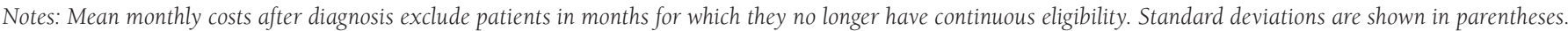
Patient counts refer to abusers.

To provide additional insights into the 3 specific underlying diagnoses constituting our definition of opioid abuse (abuse, dependence, overdose/poisoning), we also estimated excess costs within each specific index date diagnosis. Specifically, when selecting the patient sample, instead of combining patients into 1 cohort of "abusers," we instead applied the above sample selection criteria but stratified patients by their index date diagnosis. Patients with multiple abuse diagnoses were assigned to the most severe, with overdose/poisoning being the most severe, followed by abuse, then dependence.

\section{Results}

The postmatched samples were well balanced as in Kirson et al. (data on file). Figure 1 presents mean per-patient-per-month costs over the course of the study period. Health care costs increase in the 7 months before the abuse diagnosis (\$183$\$ 1,111$ per month), spike during the incident month $(\$ 6,103)$, and remain elevated in the 6 months after the initial abuse diagnosis (\$965-\$1,953 per month). Examining the extended follow-up period, we find the excess costs of abuse costs remain elevated for the year after (\$770-\$1,163 per month) as well.

The additional year of observation provides an extended view of the general composition of care received by patients over time. In the 6 months prediagnosis, the largest proportion of excess costs was incurred in the ED (40\%), followed by inpatient (28\%), outpatient (20\%), and rehabilitation facility (7\%) settings. Prescription drugs accounted for $6 \%$ of excess costs. In the first 6 months after diagnosis, changes in the composition of costs occurred that were consistent with the treatment of opioid abuse: ED costs decreased proportionally to $19 \%$ and outpatient and rehabilitation facility costs increased proportionally to 33\% and 19\%, respectively. Inpatient and prescription drug costs as percentages of excess costs remained relatively stable. These trends are similar in direction and magnitude to those observed in Kirson et al.

In the second half of the follow-up period, the composition of care shifted toward less intensive care. Inpatient costs in the 6-12 months and 12-18 months after diagnosis constituted a smaller proportion of excess costs than in the previous year; $16 \%$ and $20 \%$, respectively. ED costs remained relatively stable proportionally, $21 \%$ in months $6-12$ and $16 \%$ in months $12-18$ after diagnosis. Rehabilitation facility costs, having peaked in months 0-6 after diagnosis, were lower proportionally in the additional year (14\% in months 6-12 and $13 \%$ in months $12-18$ compared with $19 \%$ in months 0-6 after diagnosis). 
In contrast, outpatient and prescription drug costs constituted larger proportions of excess costs. Outpatient costs rose to $41 \%$ and $42 \%$ in the 6-12 months and $12-18$ months after diagnosis from $33 \%$ in months $0-6$; prescription drug costs rose to $9 \%$ and $8 \%$ in the $6-12$ and $12-18$ months after diagnosis from $4 \%$ in the first period after diagnosis.

As the definition of opioid abuse consisted of several underlying diagnoses (abuse, dependence, overdose/poisoning), we also examined excess costs by specific index date diagnosis. The majority of patients (79\%) in the abuse cohort had an index date diagnosis of opioid dependence. These patients had excess costs of $\$ 21,479$ over the 2-year follow-up period. Patients with an index date diagnosis strictly for opioid abuse (12\%) had excess costs of $\$ 17,766$ over the same period. The remaining patients (9\%) had an index date diagnosis of overdose/poisoning and had the highest excess costs at $\$ 44,173$. Within each of these 3 subgroups, we observed a similar excess cost trajectory over time: abuser and control cohorts' costs were well balanced during the baseline period; excess costs began to increase before the index date, peaked during the 6 months following the index date, and remained elevated well above baseline levels thereafter.

Table 1 presents clinical categories of medical cost drivers. The most dominant across all 4 periods were opioid abuse and nonopioid drug/alcohol abuse. Not surprisingly, given the findings in Kirson et al., the categories contributing the next largest amounts toward excess costs were painful and mental health conditions. We also observed a number of other clinical categories contributing to excess costs not observed earlier in Kirson et al. due to a more limited list of diagnosis-level cost drivers. Over the 2 years of follow-up, respiratory conditions accounted for $5 \%$ of excess costs. Gastrointestinal issues (2\%), cancers (2\%), and cardiovascular conditions (1\%) also contributed. Details on the diagnoses included in each of these categories can be found in the notes accompanying Table 1 .

\section{Discussion}

This extension study sought to provide a deeper understanding of the trajectory and drivers of the incremental costs of opioid abuse. We find that the pattern of excess costs observed in Kirson et al. persists: that is, excess costs begin accumulating before an incident abuse diagnosis, spike during the incident diagnosis month, and remain elevated above baseline levels thereafter. A contribution of this study is that the excess costs of abuse remained elevated relative to baseline levels for a full additional year. This pattern is also visible when stratifying the sample by abusers' index date diagnoses (abuse, dependence, overdose/poisoning).

These analyses provided insights into the treatment path of opioid abusers. Patients appeared to first receive more intensive care, later transitioning into ongoing follow-up care. Earlier in the follow-up period, patients incurred a sizable proportion of costs in the ED and inpatient settings, though these decreased modestly over time. Entering the second year of follow-up, the proportions of outpatient and prescription drug costs increased (33\% to $41 \%$ and $4 \%$ to $9 \%$ ) and remained at those levels. Rehabilitation costs were largely stable across the 2 years, constituting 19\% of excess costs in the first year and 14\% in the second year, perhaps due to longer-term treatment programs. That a larger proportion of costs was initially incurred in these more intensive settings (ED, inpatient) may be indicative of acute abuse-related events resulting in the formal abuse diagnoses. The subsequent trend toward maintenance care (outpatient, rehabilitation facilities) suggests patients continued to receive treatment, albeit less intensive, for their abuse-related condition.

The more detailed examination of diagnoses associated with excess costs suggests that in addition to the key areas previously identified (opioid abuse, nonopioid drug/alcohol abuse, mental health issues, and painful conditions), various respiratory, gastrointestinal, and cardiovascular diagnoses also contributed to excess costs. Since the matching algorithm adjusted for baseline characteristics, including a detailed list of comorbid conditions, these cost drivers can be reasonably interpreted as related to the opioid abuse episode.

These results suggest some important areas for future research. It is possible that opioid abuse affects the management of other medical conditions, either through a direct physiological effect, or through a more indirect effect such as reduced capacity for self-care. These are important questions for further exploration. To the extent that such an interaction effect does exist, it may suggest new avenues for early identification of abuse, and that increases in use of medical resources due to seemingly unrelated health conditions (e.g., management of cardiovascular health or diabetes) may be predictive of not-yet-diagnosed opioid abuse issues. In addition, various diagnoses that are vague in nature may turn out to be linked to increased likelihood of abuse issues. For example, fluid and electrolyte imbalances were a prominent diagnosis-level cost driver, though remained uncategorized in Table 1, and may be associated with opioid abuse. ${ }^{3}$

\section{Limitations}

This study was limited to the top 50 cost drivers within each 6 -month period. As only the top 50 cost drivers were estimated, with rankings based on excess costs, the analysis did not identify the 3-digit ICD-9-CM groupings for which excess costs were negative-that is, conditions in which nonabusers were costlier than abusers were not included in the category classifications and percent calculations. This approach results in the total percentage of cost drivers exceeding $100 \%$ (i.e., $147 \%$ ) for the 12- to 18-month period.

Additional limitations are outlined thoroughly in Kirson et al.; a brief overview follows. First, the analysis relies on the 


\begin{tabular}{|c|c|c|c|c|c|c|c|c|}
\hline \multirow[b]{2}{*}{ Total Excess Medical Costs ${ }^{\mathrm{e}}$} & \multicolumn{2}{|c|}{-6 to 0 Months } & \multicolumn{2}{|c|}{0 to 6 Months $^{\mathrm{d}}$} & \multicolumn{2}{|c|}{6 to 12 Months $^{\mathrm{d}}$} & \multicolumn{2}{|c|}{12 to 18 Months $^{d}$} \\
\hline & $\begin{array}{c}\$ 3,329 \\
{[\mathrm{SD}: \$ 26,214]} \\
(P<0.001) \\
(\$)\end{array}$ & (\%) & $\mid \begin{array}{c}\$ 11,771 \\
{[\mathrm{SD}: \$ 26,904]} \\
(P<0.001) \\
(\$)\end{array}$ & (\%) & $\begin{array}{c}\$ 3,985 \\
{[\mathrm{SD}: \$ 24,367]} \\
(P<0.001) \\
(\$)\end{array}$ & (\%) & $\begin{array}{c}\$ 2,719 \\
{[\mathrm{SD}: \$ 16,953]} \\
(P<0.001) \\
(\$)\end{array}$ & $(\%)$ \\
\hline \multicolumn{9}{|c|}{ Diagnosis-level medical cost driver groupings ${ }^{f-g}$} \\
\hline Opioid abuse & 0 & 0 & 3,815 & 32 & 1,299 & 33 & 1,182 & 43 \\
\hline Nonopioid drug and alcohol abuse ${ }^{i}$ & 1,009 & 30 & 3,559 & 30 & 1,684 & 42 & 1,742 & 64 \\
\hline Mental health conditionsj & 288 & 9 & 793 & 7 & 307 & 8 & 332 & 12 \\
\hline Painful conditionsk & 404 & 12 & 552 & 5 & 355 & 9 & 271 & 10 \\
\hline Respiratory conditions ${ }^{1}$ & 286 & 9 & 453 & 4 & 202 & 5 & 142 & 5 \\
\hline Gastrointestinal conditions ${ }^{\mathrm{m}}$ & 193 & 6 & 76 & 1 & 52 & 1 & 120 & 4 \\
\hline Cancersn & 93 & 3 & 47 & 0 & 98 & 2 & 170 & 6 \\
\hline Cardiovascular conditions $^{\circ}$ & 60 & 2 & 34 & 0 & 36 & 1 & 32 & 1 \\
\hline Total & 2,332 & 70 & 9,329 & 79 & 4,032 & 101 & 3,991 & 147 \\
\hline
\end{tabular}

aThe table reflects data for 9,345 matched pairs of abusers and controls. Abusers were matched to potential controls based on propensity score (within a quarter of a standard deviation). These counts do not reflect patient attrition in the post-index period.

${ }^{b}$ The index date for abusers was defined as the date of first diagnosis of abuse, dependence, or poisoning/overdose in 21 2012-Q1 2015. The index date for controls was defined as a random medical claim in 21 2012-Q1 2015.

cCosts were inflated to 2015 values using the medical care component of the Consumer Price Index.

${ }^{2}$ Mean costs after diagnosis shown in 6-month increments above include patients in months for which they no longer have continuous eligibility.

eP values comparing the distribution of medical costs for opioid abusers and controls were calculated using Wilcoxon signed rank tests.

fCondition categories are sorted in descending order by costs during the 0 to 6 months after diagnosis.

sTotals may exceed $100 \%$ if the top 50 diagnosis-level cost drivers excluded diagnoses for which controls' average costs exceeded those of abusers.

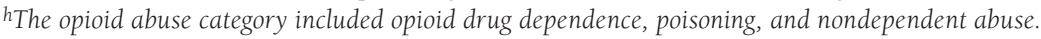

iThe nonopioid drug/alcohol abuse category contained nonopioid drug dependence, alcohol dependence syndrome, alcohol-induced mental disorders, chronic liver disease and cirrhosis, liver abscess and sequelae of chronic liver disease, nondependent abuse of nonopioid drugs, drug-induced mental disorders, and poisoning by nonopioid drugs, including antipyretics, antirheumatics, and psychotropics.

${ }^{j}$ Mental health conditions included adjustment reaction; anxiety, dissociative and somatoform disorders; depressive disorder, not elsewhere classified; episodic mood disorders; migraine; other and unspecified disorders of the nervous system; other conditions of brain; other nonorganic psychoses; other psychosocial circumstances; and trigeminal nerve disorders.

kPainful conditions included arthropathy associated with infections; atherosclerosis; curvature of spine; diffuse diseases of connective tissue; fracture of vertebral column without mention of spinal cord injury; other acquired musculoskeletal deformity; intervertebral disc disorders; osteoarthrosis and allied disorders; other and unspecified disorders of joint; other and unspecified disorders of back; other disorders of bone and cartilage; pain, not elsewhere classified; and spondylosis and allied disorders.

${ }^{l}$ Respiratory conditions included asthma; empyema; other bacterial pneumonia; other diseases of lung; pneumonia, organism unspecified; pneumonitis due to solids and liquids; and symptoms involving respiratory system and other chest symptoms.

${ }^{m}$ Gastrointestinal conditions included gastritis and duodenitis; gastrointestinal hemorrhage; intestinal infections due to other organisms; intestinal obstruction without mention of hernia; other disorders of intestine; regional enteritis; symptoms involving digestive system disorders of function of stomach; and vascular insufficiency of intestine. ${ }^{n}$ Cancers included malignant melanoma of skin; malignant neoplasm of brain; malignant neoplasm of connective and other soft tissue; malignant neoplasm of kidney and other and unspecified urinary organs; malignant neoplasm of prostate; malignant neoplasm of rectum rectosigmoid junction and anus; multiple myeloma and immunoproliferative neoplasms; and other malignant neoplasms of lymphoid and histiocytic tissue.

${ }^{\circ}$ Cardiovascular conditions included complications of bariatric procedures; other diseases of blood and blood-forming organs; other diseases of endocardium; other forms of chronic ischemic heart disease; peritonitis and retroperitoneal infections; and symptoms involving cardiovascular system.

$S D=$ standard deviation

accuracy of claims data, and any miscoding in the underlying data could affect our results, although we have no reason to believe that any inaccuracies in the data affected the abusers or nonabuser control patients differently. Second and relatedly, because undiagnosed opioid abusers do not receive any of the ICD-9-CM diagnosis codes for abuse by definition, it is possible that some of these patients may be included in our "nonabuser" cohort. While the extent to which this is the case is unknown, if undiagnosed abusers are costlier than a true "nonabuser" population, this would imply that the estimated excess costs of diagnosed abuse understate the actual excess cost differential between abusers and a control population of patients without diagnosed or undiagnosed opioid abuse. Third, propensity score matching was used to adjust for observable differences between abusers and nonabusers, but unobserved heterogeneity between cohorts may remain. Finally, our study focused on the commercially insured population, and the results herein may not generalize to other populations.

\section{Conclusions}

This study extended the follow-up period over which the excess costs of opioid abuse were measured and their cost 
drivers evaluated. Our findings align well with the previously established work and highlight that costs do not return to baseline in the 18 months after diagnosis: The incremental costs of opioid abuse in years 1 and 2 of the follow-up period were $\$ 15,764$ and $\$ 7,346$, respectively. An expanded analysis of the diagnosis-level drivers of excess costs highlighted additional potential drivers of excess costs, which may suggest areas for future research.

\section{Authors}

LAUREN M. SCARPATI, PhD; NOAM Y. KIRSON, PhD;

ZITONG B. JIA, BS; and JODY WEN, BS, Analysis Group, Boston, Massachusetts. JAREN HOWARD, PharmD, Purdue Pharma, Stamford, Connecticut.

AUTHOR CORRESPONDENCE: Lauren M. Scarpati, PhD, Analysis Group, 111 Huntington Ave., 14th Fl., Boston, MA 02199.

Tel.: 617.422.2834; E-mail: lauren.scarpati@analysisgroup.com.

\section{DISCLOSURES}

This study was funded by Purdue Pharma. Howard was an employee of Purdue Pharma at the time that this study was conducted. Kirson, Scarpati, Jia, and Wen are employees of Analysis Group, which received funding from Purdue Pharma to conduct this study.

Study concept and design were contributed by Kirson, Scarpati, and Howard, along with Jia and Wen. Jia and Wen took the lead in data collection, with assistance from Scarpati and Kirson. Data interpretation was performed by Scarpati, Kirson, and Howard, with assistance from Jia and Wen. The manuscript was written and revised by Scarpati, Kirson, and Howard.

\section{REFERENCES}

1. Kirson NY, Scarpati LM, Enloe CJ, et al. The economic burden of opioid abuse: updated findings. J Manag Care Spec Pharm. 2017;23(4):427-45. Available at: http://www.jmcp.org/doi/abs/10.18553/jmcp.2017.16265.

2. Kirson NY, Shei A, Rice JB, et al. The burden of undiagnosed opioid abuse among commercially insured individuals. Pain Medicine. 2015;16(7):1325-32.

3. Rastogi R, Swarm RA, Patel TA. Case scenario: opioid association with serotonin syndrome. Anesthesiology. 2011;115(6):1291-98. 